\title{
A PERTURBED VERSION OF GENERAL WEIGHTED OSTROWSKI TYPE INEQUALITY AND APPLICATIONS
}

\author{
WASEEM GHAZI ALSHANTI* \\ Department of General Studies, Jubail University College, Saudi Arabia
}

${ }^{*}$ Corresponding author: shantiw@ucj.edu.sa

\begin{abstract}
The main purpose of this paper is to derive some new generalizations of weighted Ostrowski type inequalities. The new established inequalities are carried out for a twice differentiable mapping in different $L_{p}$ spaces. Applications throught considering Grüss type inequality and numerical integration are also provided.
\end{abstract}

\section{Introduction}

The Ostrowski's inequality [1] can be considered as a very powerful tool for enhancement of numerical integration rules. It provides convenient potintial window for establishing bounds for the well known NewtonCotes rules. To illustrate this point, consider $f:[a, b] \rightarrow \mathbb{R}$ to be a bounded function such that $b-a$ is small, then

$$
I=\int_{a}^{b} f(x) d x
$$

can be, simply, approximated by sampling at one point as $I^{*}(x)=(b-a) f(x)$ for some $x \in[a, b]$. Now, if $f^{\prime}$ exists and is bounded, the inequality of Ostrowski may be stated as follows

$$
\left|I^{*}(x)-I\right| \leq\left[\frac{1}{4}+\left(\frac{x-\frac{a+b}{2}}{b-a}\right)^{2}\right]\left\|f^{\prime}\right\|_{\infty}
$$

Received 2018-02-12; accepted 2018-04-27; published 2018-07-02.

2010 Mathematics Subject Classification. 26D15.

Key words and phrases. Ostrowski's inequality; Weight function; Grüss inequality; Numerical integration.

(C)2018 Authors retain the copyrights of their papers, and all open access articles are distributed under the terms of the Creative Commons Attribution License. 
where

$$
\left\|f^{\prime}\right\|_{\infty}=\sup _{x \in[a, b]}\left|f^{\prime}(x)\right|
$$

Consequently, over the past few decades, there have been many studies on obtaining sharp bounds of (1.1) by considering the mappings and their derivatives in various Lebesgue spaces. Further, the new bounds have been carried out by implementing weighted and non-weighted Peano kernel. Several weighted and nonweighted versions of (1.1) have been derived. Applications in both numerical integration and probability are also presented in this regards. For instance, Roumeliotis et. al [2] proved a weighted integral inequality of Ostrowski's type for mappings whose second derivatives are bounded. Cerone [3] obtained bounds for the deviation of a function from a combination of integral means over the end intervals covering the entire interval. Qayyum et. al [4] established a new Ostrowski's type inequality using weight function which generalizes the inequality in [3]. Barnett [5] reported a companion of (1.1) and the generalized trapezoid inequalites for various classes of functions, including functions of bounded variation, Lipschitzian, convex and absolutely continuous functions. Recently, Budak et. al [6] presented a new generalization of weighted Ostrowski's type inequality for mappings of bounded variation. Several further generalizations of (1.1) are provided in [7] - [16].

In [12], Qayyum et. al proved the following non-weighted generalization of Ostrowski's type integral inequality.

Theorem 1.1. Let $f:[a, b] \rightarrow \mathbb{R}$ be a twice differentiable mapping. Then

$$
\begin{aligned}
& \mid \frac{1}{2(\alpha+\beta)}[\alpha(x-a)-\beta(b-x)] f^{\prime}(x)-f(x) \\
& +\frac{1}{\alpha+\beta}\left[\frac{\alpha}{x-a} \int_{a}^{x} f(t) d t+\frac{\beta}{b-x} \int_{x}^{b} f(t) d t\right] \mid, \\
& \leq \begin{cases}{\left[\alpha(x-a)^{2}+\beta(b-x)^{2}\right] \frac{\left\|f^{\prime \prime}\right\|_{\infty}}{6(\alpha+\beta)},} & f^{\prime \prime} \in L_{\infty}[a, b], \\
\frac{1}{(2 q+1) \frac{1}{q}}\left[\alpha^{q}(x-a)^{q+1}\right. & f^{\prime \prime} \in L_{p}[a, b], \\
\left.+\beta^{q}(b-x)^{q+1}\right] \frac{1}{q} \frac{\left\|f^{\prime \prime}\right\|_{p}}{2(\alpha+\beta)}, & p>1, \frac{1}{p}+\frac{1}{q}=1, \\
{[\alpha(x-a)+\beta(b-x)} & \\
+|\alpha(x-a)-\beta(b-x)|] \frac{\left\|f^{\prime \prime}\right\|_{1}}{4(\alpha+\beta)}, & f^{\prime \prime} \in L_{1}[a, b] .\end{cases}
\end{aligned}
$$

where $\alpha$ and $\beta$ are non-negative real numbers such that not both zero.

In this paper, motivated by the non-weighted case in [12], new general weighted Peano kernel has been defined. To obtain new general weighted inequality of Ostrowski's type that is more generalized and extended as compare to [12]. We consider a twice differentiable mapping $f$ where, respectively, $f^{\prime \prime} \in L_{\infty}, f^{\prime \prime} \in L_{p}$ 
and $f^{\prime \prime} \in L_{1}$. Moreover, we utilize Grüss type inequality to present the perturbed verion of our result. Finally, we investigate the new general weighted inequality in numerical integration.

Before we introduce our main result for a general weighted inequality of Ostrowski's type, we commence with the following definition and lemma.

Definition: Let $\omega:(a, b) \rightarrow(0, \infty)$ be a non-negative weighted function (density) such that

$$
\int_{a}^{b} \omega(t) d t<\infty
$$

The domain of $\omega$ may be finite or infinite and may vanish at the boundary points. We denote the moments

$$
\begin{aligned}
m(a, b) & =\frac{1}{b-a} \int_{a}^{b} \omega(t) d t, \quad M(a, b)=\frac{1}{b-a} \int_{a}^{b} t \omega(t) d t, \\
N(a, b) & =\frac{1}{b-a} \int_{a}^{b} t^{2} \omega(t) d t, \quad \mu(a, b)=\frac{M(a, b)}{m(a, b)}, \\
\sigma^{2}(a, b) & =\frac{N(a, b)}{m(a, b)}-\mu^{2}(a, b) .
\end{aligned}
$$

Furthermore, for a function $f:[a, b] \rightarrow \mathbb{R}$, we define the functional

$$
S(f ; a, b)=\frac{1}{b-a} \int_{a}^{b} f(t) \omega(t) d t .
$$

Lemma 1.1. Let $f:[a, b] \rightarrow \mathbb{R}$ be a twice differentiable mapping. Denote by $P_{\omega}:[a, b]^{2} \rightarrow \mathbb{R}$ the weighted Peano kernel function that is given by

$$
P_{\omega}(x, t)= \begin{cases}\frac{\alpha}{\alpha+\beta} \frac{1}{x-a} \int_{a}^{t}(t-u) \omega(u) d u, & t \in[a, x] \\ \frac{\beta}{\alpha+\beta} \frac{1}{b-x} \int_{b}^{t}(t-u) \omega(u) d u, & t \in(x, b]\end{cases}
$$

where $\alpha, \beta \geq 0$ and not both zero. Then the following identity

$$
\int_{a}^{b} P_{\omega}(x, t) f^{\prime \prime}(t) d t=\digamma(f ; \alpha, \beta),
$$

where

$$
\begin{aligned}
\digamma(f ; \alpha, \beta)= & \frac{1}{\alpha+\beta}\{[\alpha m(a, x)(x-\mu(a, x)) \\
& +\beta m(x, b)(x-\mu(x, b))] f^{\prime}(x) \\
& -[\alpha m(a, x)+\beta m(x, b)] f(x) \\
& +\alpha S(f ; a, x)+\beta S(f ; x, b)\},
\end{aligned}
$$

holds. 
Proof: From (1.5), we have

$$
\begin{aligned}
& \int_{a}^{b} P_{\omega}(x, t) f^{\prime \prime}(t) d t \\
= & \frac{\alpha}{\alpha+\beta} \frac{1}{x-a} \int_{a}^{x}\left(\int_{a}^{t}(t-u) \omega(u) d u\right) f^{\prime \prime}(t) d t \\
& +\frac{\beta}{\alpha+\beta} \frac{1}{b-x} \int_{x}^{b}\left(\int_{b}^{t}(t-u) \omega(u) d u\right) f^{\prime \prime}(t) d t \\
= & \frac{\alpha}{\alpha+\beta} \frac{1}{x-a}\left[f^{\prime}(x) \int_{a}^{x}(x-u) \omega(u) d u\right. \\
& \left.-f(x) \int_{a}^{x} \omega(u) d u+\int_{a}^{x} f(t) \omega(t) d t\right] \\
+ & \frac{\beta}{\alpha+\beta} \frac{1}{b-x}\left[f^{\prime}(x) \int_{x}^{b}(x-u) \omega(u) d u\right. \\
& \left.-f(x) \int_{b}^{x} \omega(u) d u+\int_{x}^{b} f(t) \omega(t) d t\right] .
\end{aligned}
$$

After further simplification, the identity (1.6) can be obtained.

\section{Main Results}

Theorem 2.1. Let $f:[a, b] \rightarrow \mathbb{R}$ be a twice differentiable mapping in $(a, b)$. Then $\forall x \in[a, b]$, we have

$$
\begin{aligned}
& |\digamma(f ; \alpha, \beta)| \\
& \left\{\begin{array}{l}
\left\{\alpha m(a, x)\left[(x-\mu(a, x))^{2}+\sigma^{2}(a, x)\right]\right. \\
\left.+\beta m(x, b)\left[(x-\mu(x, b))^{2}+\sigma^{2}(x, b)\right]\right\} \quad f^{\prime \prime} \in L_{\infty}[a, b], \\
\times \frac{\left\|f^{\prime \prime}\right\|_{\infty}}{2(\alpha+\beta)},
\end{array}\right. \\
& \leq \begin{cases}{\left[\frac{\alpha^{q}}{(x-a)^{q}} \int_{a}^{x}(t-a)^{2 q} m^{q}(a, t) d t\right.} & \\
\left.+\frac{\beta^{q}}{(b-x)^{q}} \int_{x}^{b}(b-t)^{2 q} m^{q}(t, b) d t\right]^{\prime \prime} \frac{\| f_{p}[a, b],}{2(\alpha+\beta)}, & p>1, \frac{1}{p}+\frac{1}{q}=1,\end{cases} \\
& \max \{\alpha m(a, x)(x-\mu(a, x)), \\
& \beta m(x, b)(\mu(x, b)-x)\} \frac{\left\|f^{\prime \prime}\right\|_{1}}{\alpha+\beta}, \quad f^{\prime \prime} \in L_{1}[a, b] .
\end{aligned}
$$

where the functional $\digamma(f ; \alpha, \beta)$ is defined in $(1.6)$. 
Proof: Taking the modulus of the right hand side of (1.6), yields

$$
\left|\int_{a}^{b} P_{\omega}(x, t) f^{\prime \prime}(t) d t\right| \leq \int_{a}^{b}\left|P_{\omega}(x, t)\right|\left|f^{\prime \prime}(t)\right| d t .
$$

Hence, for $f^{\prime \prime} \in L_{\infty}[a, b]$

$$
|\digamma(f ; \alpha, \beta)| \leq\left\|f^{\prime \prime}\right\|_{\infty} \int_{a}^{b}\left|P_{\omega}(x, t)\right| d t .
$$

Now, using (1.5) provids,

$$
\begin{aligned}
& \int_{a}^{b}\left|P_{\omega}(x, t)\right| d t \\
& =\frac{\alpha}{\alpha+\beta} \frac{1}{x-a}\left[\frac{x^{2}}{2} \int_{a}^{x} \omega(u) d u\right. \\
& \left.-x \int_{a}^{x} u \omega(u) d u+\frac{1}{2} \int_{a}^{x} t^{2} \omega(t) d t\right] \\
& +\frac{\beta}{\alpha+\beta} \frac{1}{b-x}\left[\frac{x^{2}}{2} \int_{x}^{b} \omega(u) d u\right. \\
& \left.-x \int_{x}^{b} u \omega(u) d u+\frac{1}{2} \int_{x}^{b} t^{2} \omega(t) d t\right] .
\end{aligned}
$$

Thus, by combining (2.3) and (2.4), the first inequality of (2.1) results.

Further, from (2.2) and by using Hölder's integral inequality for $f^{\prime \prime} \in L_{p}[a, b]$, we have

$$
|\digamma(f ; \alpha, \beta)| \leq\left\|f^{\prime \prime}\right\|_{p}\left(\int_{a}^{b}\left|P_{\omega}(x, t)\right|^{q} d t\right)^{\frac{1}{q}}
$$

where $\frac{1}{p}+\frac{1}{q}=1$ with $p>1$. Now, by (1.5) and utilizing the weighted mean value theorem for integrals, we have

$$
\begin{gathered}
\int_{a}^{b}\left|P_{\omega}(x, t)\right|^{q} d t \\
=\left(\frac{\alpha}{\alpha+\beta}\right)^{q} \frac{1}{(x-a)^{q}} \int_{a}^{x}\left(\int_{a}^{t}(t-u) \omega(u) d u\right)^{q} d t
\end{gathered}
$$




$$
\begin{aligned}
& +\left(\frac{\beta}{\alpha+\beta}\right)^{q} \frac{1}{(b-x)^{q}} \int_{x}^{b}\left(\int_{b}^{t}(t-u) \omega(u) d u\right)^{q} d t \\
= & \left(\frac{\alpha}{\alpha+\beta}\right)^{q} \frac{1}{(x-a)^{q}} \int_{a}^{x}\left(\frac{t-a}{2} \int_{a}^{t} \omega(u) d u\right)^{q} d t \\
& +\left(\frac{\beta}{\alpha+\beta}\right)^{q} \frac{1}{(b-x)^{q}} \int_{x}^{b}\left(\frac{b-t}{2} \int_{t}^{b} \omega(u) d u\right)^{q} d t,
\end{aligned}
$$

and so, by considering (2.5) and (2.6) the second inequality of (2.1) is obtained.

Finally, for $f^{\prime \prime} \in L_{1}[a, b]$, we have from (1.6)

$$
\digamma(f ; \alpha, \beta) \leq \sup _{t \in[a, b]}\left|P_{\omega}(x, t)\right|\left\|f^{\prime \prime}\right\|_{1},
$$

where,

$$
\begin{aligned}
\sup _{t \in[a, b]}\left|P_{\omega}(x, t)\right|= & \max \left\{\frac{\alpha}{\alpha+\beta} \frac{1}{x-a} \int_{a}^{x}(x-u) \omega(u) d u\right. \\
& \left.\frac{\beta}{\alpha+\beta} \frac{1}{b-x} \int_{x}^{b}(u-x) \omega(u) d u\right\} \\
= & \frac{1}{\alpha+\beta} \max \{\alpha m(a, x)(x-\mu(a, x) \\
& \beta m(x, b)(\mu(x, b)-x]\}
\end{aligned}
$$

Therefore, combining (2.7) and (2.8) gives the third inequality of (2.1), and so, the theorem is now completely proven.

Remark 2.1. Setting $\omega(u)=1$ in Theorem (2.1) provids the corresponding non-weighted result (1.2) in [12]. For different weights, a variety of results can be obtained. 
Corollary 2.1. Let the condition of Theorem (2.1) holds. Then

$$
\begin{aligned}
& |\digamma(f ; \alpha, \beta)| \\
\leq & \left\{\alpha m(a, x)\left[(x-\mu(a, x))^{2}+\sigma^{2}(a, x)\right]\right. \\
& \left.+\beta m(x, b)\left[(x-\mu(x, b))^{2}+\sigma^{2}(x, b)\right]\right\} \\
& \times \frac{\left\|f^{\prime \prime}\right\|_{\infty}}{2(\alpha+\beta)} \\
\leq & {\left[\alpha(x-a)^{2} m(a, x)+\beta(x-b)^{2} m(x, b)\right] } \\
& \times \frac{\left\|f^{\prime \prime}\right\|_{\infty}}{2(\alpha+\beta)} .
\end{aligned}
$$

Proof: From (1.5), we have

$$
\begin{aligned}
& \int_{a}^{b}\left|P_{\omega}(x, t)\right| d t \\
= & \frac{1}{2} \frac{\alpha}{\alpha+\beta} \frac{1}{x-a} \int_{a}^{x}(x-t)^{2} \omega(t) d t \\
& +\frac{1}{2} \frac{\beta}{\alpha+\beta} \frac{1}{b-x} \int_{x}^{b}(x-t)^{2} \omega(t) d t .
\end{aligned}
$$

Now, by noting that

$$
\begin{aligned}
\int_{a}^{x}(x-t)^{2} \omega(t) d t & \leq \sup _{t \in[a, x]}(x-t)^{2}[(x-a) m(a, x)] \\
& =(x-a)^{3} m(a, x)
\end{aligned}
$$

and

$$
\begin{aligned}
\int_{x}^{b}(x-t)^{2} \omega(t) d t & \leq \sup _{t \in(x, b]}(x-t)^{2}[(b-x) m(x, b)] \\
& =(b-x)^{3} m(x, b)
\end{aligned}
$$

The desired second inequality of (2.9) can be obtained.

Corollary 2.2. Setting $\alpha=\beta$ in Theorem (2.1) gives

$$
\begin{aligned}
& \mid \frac{1}{2}\left\{[m(a, x)(x-\mu(a, x))+m(x, b)(x-\mu(x, b))] f^{\prime}(x)\right. \\
& -(m(a, x)+m(x, b)) f(x)+S(f ; a, x)+S(f ; x, b)\} \mid
\end{aligned}
$$




$$
\leq \begin{cases}\left\{m(a, x)\left[(x-\mu(a, x))^{2}+\sigma^{2}(a, x)\right]\right. & \\ \left.+m(x, b)\left[(x-\mu(x, b))^{2}+\sigma^{2}(x, b)\right]\right\} & f^{\prime \prime} \in L_{\infty}[a, b], \\ \times \frac{\left\|f^{\prime \prime}\right\|_{\infty}}{4}, & \\ {\left[\frac{1}{(x-a)^{q}} \int_{a}^{x}(t-a)^{2 q} m^{q}(a, t) d t\right.} & \\ \left.+\frac{1}{(b-x)^{q}} \int_{x}^{b}(b-t)^{2 q} m^{q}(t, b) d t\right]^{\frac{1}{q}} \frac{\left\|f^{\prime \prime}\right\|_{p}}{4}, & p>1, \frac{1}{p}+\frac{1}{q}=1, \\ & \\ \max \{m(a, x)(x-\mu(a, x)), & \\ m(x, b)(\mu(x, b)-x)\} \frac{\left\|f^{\prime \prime}\right\|_{1}}{2}, & f^{\prime \prime} \in L_{1}[a, b] .\end{cases}
$$

Remark 2.2. Setting $\omega(u)=1$ in Corollary (2.2) gives the corresponding non-weighted result obtained by [12] for the case $\alpha=\beta$.

Corollary 2.3. Setting $x=(a+b) / 2$ in Theorem (2.1) gives

$$
\begin{aligned}
& \mid \frac{1}{\alpha+\beta}\left\{\left[\alpha m\left(a, \frac{a+b}{2}\right)\left(\frac{a+b}{2}-\mu\left(a, \frac{a+b}{2}\right)\right)\right.\right. \\
& \left.+\beta m\left(\frac{a+b}{2}, b\right)\left(\frac{a+b}{2}-\mu\left(\frac{a+b}{2}, b\right)\right)\right] f^{\prime}\left(\frac{a+b}{2}\right) \\
& -\left[\alpha m\left(a, \frac{a+b}{2}\right)+\beta m\left(\frac{a+b}{2}, b\right)\right] f\left(\frac{a+b}{2}\right) \\
& \left.+\alpha S\left(f ; a, \frac{a+b}{2}\right)+\beta S\left(f ; \frac{a+b}{2}, b\right)\right\} \mid \\
& \left\{\begin{array}{l}
\left\{\alpha m\left(a, \frac{a+b}{2}\right)\left[\left(\frac{a+b}{2}-\mu\left(a, \frac{a+b}{2}\right)\right)^{2}+\sigma^{2}\left(a, \frac{a+b}{2}\right)\right]\right. \\
\left.+\beta m\left(\frac{a+b}{2}, b\right)\left[\left(\frac{a+b}{2}-\mu\left(\frac{a+b}{2}, b\right)\right)^{2}+\sigma^{2}\left(\frac{a+b}{2}, b\right)\right]\right\} \quad f^{\prime \prime} \in L_{\infty}[a, b], \\
\times\left\|f^{\prime \prime}\right\|_{\infty},
\end{array}\right. \\
& \leq\left\{\left[\alpha^{q} \int_{a}^{\frac{a+b}{2}}(t-a)^{2 q} m^{q}(a, t) d t\right.\right. \\
& \left.+\beta^{q} \int_{\frac{a+b}{2}}^{b}(b-t)^{2 q} m^{q}(t, b) d t\right]^{\frac{1}{q}} \quad \begin{array}{ll}
f^{\prime \prime} \in L_{p}[a, b], \\
\frac{\left\|f^{\prime \prime}\right\|_{p}}{(b-a)(\alpha+\beta)}, & p>1, \frac{1}{p}+\frac{1}{q}=1,
\end{array} \\
& \max \left\{\alpha m\left(a, \frac{a+b}{2}\right)\left(\frac{a+b}{2}-\mu\left(a, \frac{a+b}{2}\right)\right),\right. \\
& \left.\beta m\left(\frac{a+b}{2}, b\right)\left(\mu\left(\frac{a+b}{2}, b\right)-\frac{a+b}{2}\right)\right\} \frac{\left\|f^{\prime \prime}\right\|_{1}}{\alpha+\beta}, \quad f^{\prime \prime} \in L_{1}[a, b] \text {. }
\end{aligned}
$$


Remark 2.3. Setting $\omega(u)=1$ in Corollary (2.3), gives the corresponding non-weighted result obtained by [12] when $x=(a+b) / 2$.

Corollary 2.4. If (2.10) evaluated at $x=(a+b) / 2$, then

$$
\begin{aligned}
& \mid \frac{1}{2}\left\{\left[m\left(a, \frac{a+b}{2}\right)\left(\frac{a+b}{2}-\mu\left(a, \frac{a+b}{2}\right)\right)\right.\right. \\
& \left.+m\left(\frac{a+b}{2}, b\right)\left(x-\mu\left(\frac{a+b}{2}, b\right)\right)\right] f^{\prime}\left(\frac{a+b}{2}\right) \\
& -\left(m\left(a, \frac{a+b}{2}\right)+m\left(\frac{a+b}{2}, b\right)\right) f\left(\frac{a+b}{2}\right) \\
& \left.+S\left(f ; a, \frac{a+b}{2}\right)+S\left(f ; \frac{a+b}{2}, b\right)\right\} \mid \\
& \left\{\begin{array}{l}
\left\{m\left(a, \frac{a+b}{2}\right)\left[\left(\frac{a+b}{2}-\mu\left(a, \frac{a+b}{2}\right)\right)^{2}+\sigma^{2}\left(a, \frac{a+b}{2}\right)\right]\right. \\
\left.+m\left(\frac{a+b}{2}, b\right)\left[\left(\frac{a+b}{2}-\mu\left(\frac{a+b}{2}, b\right)\right)^{2}+\sigma^{2}\left(\frac{a+b}{2}, b\right)\right]\right\}
\end{array}\right. \\
& \times \frac{\left\|f^{\prime \prime}\right\|_{\infty}}{4} \text {, } \\
& f^{\prime \prime} \in L_{\infty}[a, b], \\
& {\left[\int_{a}^{\frac{a+b}{2}}(t-a)^{2 q} m^{q}(a, t) d t\right.}
\end{aligned}
$$

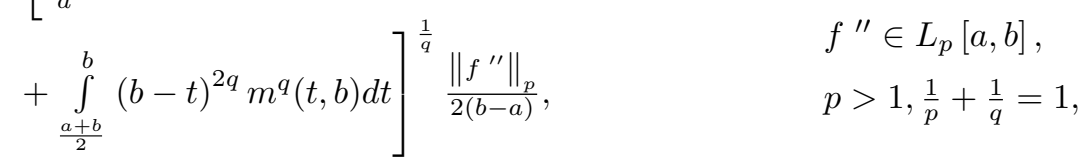

$$
\begin{aligned}
& \max \left\{m\left(a, \frac{a+b}{2}\right)\left(\frac{a+b}{2}-\mu\left(a, \frac{a+b}{2}\right)\right),\right. \\
& \left.m\left(\frac{a+b}{2}, b\right)\left(\mu\left(\frac{a+b}{2}, b\right)-\frac{a+b}{2}\right)\right\} \frac{\left\|f^{\prime \prime}\right\|_{1}}{2}, \quad f^{\prime \prime} \in L_{1}[a, b] .
\end{aligned}
$$

\section{Perturbed Results}

The Grüss inequality is as follows [13].

Theorem 3.1. Let $f, g:[a, b] \rightarrow \mathbb{R}$ be integrable functions on $[a, b]$ such that $\varphi \leq f(x) \leq \Phi$ and $\gamma \leq g(x) \leq \Gamma$ $\forall x \in[a, b]$, where $\varphi, \Phi, \gamma, \Gamma$ are constants. Then

$$
\begin{aligned}
& \left|\frac{1}{b-a} \int_{a}^{b} f(t) g(t) d t-\frac{1}{b-a} \int_{a}^{b} f(t) d t \cdot \frac{1}{b-a} \int_{a}^{b} f(t) d t\right| \\
\leq & \frac{1}{4}(\Phi-\varphi)(\Gamma-\gamma),
\end{aligned}
$$

where the constant $\frac{1}{4}$ is sharp.

Now, the perturbed verions of the results in the pervious section may be obtained by using Grüss type inequalities involving the Čebyŝev functional [14],

$$
T(f, g)=M(f g ; a, b)-M(f ; a, b) M(g ; a, b),
$$


where

$$
M(f ; a, b)=\frac{1}{b-a} \int_{a}^{b} f(t) d t,
$$

is the integral mean of $f$ over $[a, b]$.

Theorem 3.2. Let $f:[a, b] \rightarrow \mathbb{R}$ be a twice differentiable mapping such that $\gamma \leq f^{\prime \prime}(x) \leq \Gamma \forall x \in[a, b]$ and $\alpha \geq 0, \beta \geq 0, \alpha+\beta \neq 0$. Then

$$
\begin{aligned}
& \mid \digamma(f ; \alpha, \beta)-\frac{k}{2(\alpha+\beta)}\left[\alpha m(a, x)\left[(x-\mu(a, x))^{2}+\sigma^{2}(a, x)\right]\right. \\
& \left.+\beta m(x, b)\left[(x-\mu(x, b))^{2}+\sigma^{2}(x, b)\right]\right] \mid \\
\leq & (b-a) N(x)\left[\frac{\left\|f^{\prime \prime}\right\|_{2}^{2}}{b-a}-k^{2}\right]^{\frac{1}{2}}, \\
\leq & (b-a) N(x) \frac{\Gamma-\gamma}{2}, \\
\leq & \frac{(b-a)(\Gamma-\gamma)}{4(\alpha+\beta)} \max \{\alpha m(a, x)(x-\mu(a, x)), \\
& \beta m(x, b)(\mu(x, b)-x)\}
\end{aligned}
$$

where $\digamma(f ; \alpha, \beta)$ is given by $(1.7), k=\left(f^{\prime}(b)-f^{\prime}(a)\right) /(b-a)$, and

$$
\begin{aligned}
N(x)= & \left\{\left[\left(\frac{\alpha}{x-a}\right)^{2} \int_{a}^{x}(t-a)^{4} m^{2}(a, t) d t\right.\right. \\
& \left.+\left(\frac{\beta}{b-x}\right)^{2} \int_{x}^{b}(b-t)^{4} m^{2}(t, b) d t\right] \frac{1}{4(\alpha+\beta)^{2}(b-a)} \\
& -\left\{\left[\alpha m(a, x)\left[(x-\mu(a, x))^{2}+\sigma^{2}(a, x)\right]\right.\right. \\
& \left.\left.\left.+\beta m(x, b)\left[(x-\mu(x, b))^{2}+\sigma^{2}(x, b)\right]\right] \frac{1}{2(\alpha+\beta)(b-a)}\right\}^{2}\right\}^{\frac{1}{2}} .
\end{aligned}
$$

Proof: Replacing $f(t)$ by $P_{\omega}(x, t)$ and $g(t)$ by $f^{\prime \prime}(x)$ in (3.2) yields,

$$
\begin{aligned}
T\left(P_{\omega}(x, t), f^{\prime \prime}(x)\right)= & M\left(P_{\omega}(x, t) f^{\prime \prime}(x) ; a, b\right) \\
& -M\left(P_{\omega}(x, t) ; a, b\right) M\left(f^{\prime \prime}(x) ; a, b\right) .
\end{aligned}
$$

Now, by using both (1.6) and (2.4), we have

$$
\begin{aligned}
T\left(P_{\omega}(x, t), f^{\prime \prime}(x)\right)= & \frac{1}{b-a}\{\digamma(f ; \alpha, \beta) \\
& -\frac{k}{2(\alpha+\beta)}\left[\alpha m(a, x)\left[(x-\mu(a, x))^{2}+\sigma^{2}(a, x)\right]\right. \\
& \left.\left.+\beta m(x, b)\left[(x-\mu(x, b))^{2}+\sigma^{2}(x, b)\right]\right]\right\},
\end{aligned}
$$


where $k$ is the secant slope of $f^{\prime}$ over $[a, b]$ as given in (3.4). Moreover, by [3], we have

$$
\begin{aligned}
T\left(P_{\omega}(x, t), f^{\prime \prime}(x)\right) \leq & T^{\frac{1}{2}}\left(P_{\omega}(x, t), P_{\omega}(x, t)\right) T^{\frac{1}{2}}\left(f^{\prime \prime}(x), f^{\prime \prime}(x)\right), \\
& \left(P_{\omega}(x, t), f^{\prime \prime}(x) \in L_{2}[a, b]\right) \\
\leq & \frac{\Gamma-\gamma}{2} T^{\frac{1}{2}}\left(P_{\omega}(x, t), P_{\omega}(x, t)\right), \\
& \left(\gamma \leq f^{\prime \prime}(x) \leq \Gamma, \forall x \in[a, b]\right) \\
\leq & \frac{1}{4}(\Phi-\varphi)(\Gamma-\gamma), \\
& \left(\varphi \leq P_{\omega}(x, t) \leq \Phi, \forall(x, t) \in[a, b]^{2}\right) .
\end{aligned}
$$

But,

$$
\begin{aligned}
0 & \leq T^{\frac{1}{2}}\left(f^{\prime \prime}(x), f^{\prime \prime}(x)\right) \\
& =\left[M\left(\left(f^{\prime \prime}(x)\right)^{2} ; a, b\right)-M^{2}\left(f^{\prime \prime}(x) ; a, b\right)\right]^{\frac{1}{2}} \\
& =\left[\frac{1}{b-a} \int_{a}^{b}\left(f^{\prime \prime}(x)\right)^{2} d x-\left(\frac{\int_{a}^{b} f^{\prime \prime}(x) d x}{b-a}\right)^{2}\right]^{\frac{1}{2}} \\
& =\left[\frac{1}{b-a}\left\|f^{\prime \prime}\right\|_{2}^{2}-k^{2}\right]^{\frac{1}{2}} \\
& \leq \frac{\Gamma-\gamma}{2},
\end{aligned}
$$

where $\gamma \leq f^{\prime \prime}(x) \leq \Gamma, \forall x \in[a, b]$.

Now, for $T^{\frac{1}{2}}\left(P_{\omega}(x, t), P_{\omega}(x, t)\right)$, we consider (1.5) as follows

$$
\begin{aligned}
0 & \leq T^{\frac{1}{2}}\left(P_{\omega}(x, t), P_{\omega}(x, t)\right) \\
& =\left[M\left(\left(P_{\omega}(x, t)\right)^{2} ; a, b\right)-M^{2}\left(P_{\omega}(x, t) ; a, b\right)\right]^{\frac{1}{2}} \\
& =\left[\frac{1}{b-a} \int_{a}^{b}\left(P_{\omega}(x, t)\right)^{2} d x-\left(\frac{\int_{a}^{b} P_{\omega}(x, t) d x}{b-a}\right)^{2}\right]^{\frac{1}{2}} \\
& =N(x),
\end{aligned}
$$

where $N(x)$ is given in (3.4) .

Therefore, by combining (3.6), (3.7), (3.8), and (3.9) gives the first and the second inequalities of (3.3). Further, to determine the values of $\varphi$ and $\Phi$ for which $\varphi \leq P_{\omega}(x, t) \leq \Phi, \forall(x, t) \in[a, b]^{2}$, it may be noticed 
from the definition of $P_{\omega}(x, t)$ in (1.5) that for $\alpha, \beta \geq 0, \alpha+\beta \neq 0$, we have

$$
\begin{aligned}
\Phi & =\sup _{t \in[a, b]} P_{\omega}(x, t) \\
& =\frac{1}{\alpha+\beta} \max \{\alpha m(a, x)(x-\mu(a, x)), \beta m(x, b)(\mu(x, b)-x)\}, \\
\varphi & =\inf _{t \in[a, b]} P_{\omega}(x, t)=0 .
\end{aligned}
$$

Hence, from (3.6), (3.8), (3.10) and the last inequality in (3.7), we obtain the third inequality in (3.3) and the theorem is now completely proved.

\section{Application in Numerical Integration}

Let $a=\zeta_{\circ}<\zeta_{1}<\cdots<\zeta_{n-1}<\zeta_{n}=x=\eta_{\circ}<\eta_{1}<\cdots<\eta_{n-1}<\eta_{n}=b$ be a partition of the interval $[a, b]$, with $x_{i} \in\left[\zeta_{i}, \zeta_{i+1}\right]$ for $i=0,1, \ldots . ., n-1, x_{j}^{*} \in\left[\eta_{j}, \eta_{j+1}\right]$ for $j=0,1, \ldots ., n-1, \delta=\zeta_{i+1}-\zeta_{i}$, and $\Delta=\eta_{j+1}-\eta_{j}$. Consider the following general quadrature rule.

$$
\begin{aligned}
& A(f, \zeta, \eta, x) \\
= & \alpha \sum_{i=0}^{n-1} m_{i}\left[f\left(x_{i}\right)-\left(x_{i}-\mu_{i}\right) f^{\prime}\left(x_{i}\right)\right] \\
& +\beta \sum_{j=0}^{n-1} m_{j}^{*}\left[f\left(x_{j}^{*}\right)-\left(x_{j}^{*}-\mu_{j}^{*}\right) f^{\prime}\left(x_{j}^{*}\right)\right] .
\end{aligned}
$$

Theorem 4.1. Let the conditions of Theorem (2.1) hold. The following weighted quadrature rule for weighted integral holds

$$
\begin{aligned}
& \frac{\alpha}{\delta} \int_{a}^{x} f(t) \omega(t) d t+\frac{\beta}{\Delta} \int_{x}^{b} f(t) \omega(t) d t \\
= & A(f, \zeta, \eta, x)+R(f, \zeta, \eta, x),
\end{aligned}
$$

where $A(f, \zeta, \eta, x)$ is defined by (4.1), the remainder $R(f, \zeta, \eta, x)$ satisfies the estimate

$$
\begin{aligned}
& R(f, \zeta, \eta, x) \\
\leq & \left\{\alpha \sum_{i=0}^{n-1} m_{i}\left[\left(x_{i}-\mu_{i}\right)^{2}+\sigma_{i}^{2}\right]\right. \\
+ & \left.\beta \sum_{j=0}^{n-1} m_{j}^{*}\left[\left(x_{j}^{*}-\mu_{j}^{*}\right)^{2}+\sigma_{j}^{* 2}\right]\right\} \frac{\left\|f^{\prime \prime}\right\|_{\infty}}{2},
\end{aligned}
$$

and the parameters $m_{i}, \mu_{i}, \sigma_{i}^{2}, m_{j}^{*}, \mu_{j}^{*}$, and $\sigma_{j}^{* 2}$ are given by

$$
\begin{aligned}
& m_{i}=m\left(\zeta_{i}, \zeta_{i+1}\right), \quad \mu_{i}=\mu\left(\zeta_{i}, \zeta_{i+1}\right), \quad \sigma_{i}^{2}=\sigma^{2}\left(\zeta_{i}, \zeta_{i+1}\right), \\
& m_{j}^{*}=m\left(\eta_{j}, \eta_{j+1}\right), \quad \mu_{j}^{*}=\mu\left(\eta_{j}, \eta_{j+1}\right), \quad \text { and } \sigma_{j}^{* 2}=\sigma^{2}\left(\eta_{j}, \eta_{j+1}\right) .
\end{aligned}
$$


Proof: Applying the first inequality of (2.1) over the interval $\left[\zeta_{i}, \zeta_{i+1}\right]$ with $x=x_{i} \in\left[\zeta_{i}, \zeta_{i+1}\right]$ and over the interval $\left[\eta_{j}, \eta_{j+1}\right]$ with $x=x_{j}^{*} \in\left[\eta_{j}, \eta_{j+1}\right]$ gives

$$
\begin{aligned}
& \mid \alpha m_{i}\left(x_{i}-\mu_{i}\right) f^{\prime}\left(x_{i}\right)+\beta m_{j}^{*}\left(x_{j}^{*}-\mu_{j}^{*}\right) f^{\prime}\left(x_{j}^{*}\right)-\alpha m_{i} f\left(x_{i}\right)+\beta m_{j}^{*} f\left(x_{j}^{*}\right) \\
& +\frac{\alpha}{\delta} \int_{\zeta_{i}}^{\zeta_{i+1}} f(t) \omega(t) d t+\frac{\beta}{\Delta} \int_{\eta_{j}}^{\eta_{j+1}} f(t) \omega(t) d t \mid \\
\leq & \left\{\alpha m_{i}\left[\left(x_{i}-\mu_{i}\right)^{2}+\sigma_{i}^{2}\right]+\beta m_{j}^{*}\left[\left(x_{j}^{*}-\mu_{j}^{*}\right)^{2}+\sigma_{j}^{* 2}\right]\right\} \frac{\left\|f^{\prime \prime}\right\|_{\infty}}{2},
\end{aligned}
$$

for all $i, j=0,1, \ldots . ., n-1$. Summing over $i, j$ from 0 to $n-1$ and using the triangle inequality produces the desired result (4.2).

Theorem 4.2. Let the conditions of Theorem (2.1) hold. The following weighted quadrature rule for weighted integral holds

$$
\begin{aligned}
& \frac{\alpha}{\delta} \int_{a}^{x} f(t) \omega(t) d t+\frac{\beta}{\Delta} \int_{x}^{b} f(t) \omega(t) d t \\
= & A(f, \zeta, \eta, x)+R(f, \zeta, \eta, x),
\end{aligned}
$$

where $A(f, \zeta, \eta, x)$ is defined by (4.1), the remainder $R(f, \zeta, \eta, x)$ satisfies the estimate

$$
\begin{aligned}
& R(f, \zeta, \eta, x) \\
\leq & {\left[\frac{\alpha^{q}}{\delta^{q}} \int_{\zeta_{i}}^{\zeta_{i+1}}\left(t-\zeta_{i}\right)^{2 q} m^{q}\left(\zeta_{i}, t\right) d t\right.} \\
& \left.+\frac{\beta^{q}}{\Delta^{q}} \int_{\eta_{j}}^{\eta_{j+1}}\left(\eta_{j+1}-t\right)^{2 q} m^{q}\left(t, \eta_{j+1}\right) d t\right]^{\frac{1}{q}} \frac{\left\|f^{\prime \prime}\right\|_{p}}{2} .
\end{aligned}
$$

Proof: Applying the second inequality of (2.1) over the interval $\left[\zeta_{i}, \zeta_{i+1}\right]$ with $x=x_{i} \in\left[\zeta_{i}, \zeta_{i+1}\right]$ and over the interval $\left[\eta_{j}, \eta_{j+1}\right]$ with $x=x_{j}^{*} \in\left[\eta_{j}, \eta_{j+1}\right]$ gives

$$
\begin{aligned}
& \quad \mid \alpha m_{i}\left(x_{i}-\mu_{i}\right) f^{\prime}\left(x_{i}\right)+\beta m_{j}^{*}\left(x_{j}^{*}-\mu_{j}^{*}\right) f^{\prime}\left(x_{j}^{*}\right)-\alpha m_{i} f\left(x_{i}\right)+\beta m_{j}^{*} f\left(x_{j}^{*}\right) \\
& +\frac{\alpha}{\delta} \int_{\zeta_{i}}^{\zeta_{i+1}} f(t) \omega(t) d t+\frac{\beta}{\Delta} \int_{\eta_{j}}^{\eta_{j+1}} f(t) \omega(t) d t \mid \\
& \leq \quad\left[\frac{\alpha^{q}}{\delta^{q}} \int_{\zeta_{i}}^{\zeta_{i+1}}\left(t-\zeta_{i}\right)^{2 q} m^{q}\left(\zeta_{i}, t\right) d t\right. \\
& \left.+\frac{\beta^{q}}{\Delta^{q}} \int_{\eta_{j}}^{\eta_{j+1}}\left(\eta_{j+1}-t\right)^{2 q} m^{q}\left(t, \eta_{j+1}\right) d t\right]^{\frac{1}{q}} \frac{\left\|f^{\prime \prime}\right\|_{p}}{2} .
\end{aligned}
$$


for all $i, j=0,1, \ldots ., n-1$. Summing over $i, j$ from 0 to $n-1$ and using the triangle inequality produces the desired result (4.5).

Theorem 4.3. Let the conditions of Theorem (2.1) hold. The following weighted quadrature rule for weighted integral holds

$$
\begin{aligned}
& \frac{\alpha}{\delta} \int_{a}^{x} f(t) \omega(t) d t+\frac{\beta}{\Delta} \int_{x}^{b} f(t) \omega(t) d t \\
= & A(f, \zeta, \eta, x)+R(f, \zeta, \eta, x),
\end{aligned}
$$

where $A(f, \zeta, \eta, x)$ is defined by (4.1), the remainder $R(f, \zeta, \eta, x)$ satisfies the estimate

$$
\begin{aligned}
& R(f, \zeta, \eta, x) \\
\leq & \max \left[\alpha m_{i}\left(x_{i}-\mu_{i}\right), \beta m_{j}^{*}\left(\mu_{j}^{*}-x_{j}^{*}\right)\right]\left\|f^{\prime \prime}\right\|_{1} .
\end{aligned}
$$

Proof: Applying the third inequality of (2.1) over the interval $\left[\zeta_{i}, \zeta_{i+1}\right]$ with $x=x_{i} \in\left[\zeta_{i}, \zeta_{i+1}\right]$ and over the interval $\left[\eta_{j}, \eta_{j+1}\right]$ with $x=x_{j}^{*} \in\left[\eta_{j}, \eta_{j+1}\right]$ gives

$$
\begin{aligned}
& \mid \alpha m_{i}\left(x_{i}-\mu_{i}\right) f^{\prime}\left(x_{i}\right)+\beta m_{j}^{*}\left(x_{j}^{*}-\mu_{j}^{*}\right) f^{\prime}\left(x_{j}^{*}\right)-\alpha m_{i} f\left(x_{i}\right)+\beta m_{j}^{*} f\left(x_{j}^{*}\right) \\
& +\frac{\alpha}{\delta} \int_{\zeta_{i}}^{\zeta_{i+1}} f(t) \omega(t) d t+\frac{\beta}{\Delta} \int_{\eta_{j}}^{\eta_{j+1}} f(t) \omega(t) d t \mid \\
& \leq \max \left\{\alpha m_{i}\left(x_{i}-\mu_{i}\right), \beta m_{j}^{*}\left(\mu_{j}^{*}-x_{j}^{*}\right)\right\}\left\|f^{\prime \prime}\right\|_{1} .
\end{aligned}
$$

for all $i, j=0,1, \ldots ., n-1$. Summing over $i, j$ from 0 to $n-1$ and using the triangle inequality produces the desired result (4.6).

\section{REFERENCES}

[1] A. Ostrowski, Über die Absolutabweichung einer differentienbaren Funktionen von ihren Integralimittelwert, Comment. Math. Hel. 10 (1938), 226-227.

[2] J. Roumeliotis, P. Cerone, and S. S. Dragomir, An Ostrowski type inequality for weighted mappings with bounded second derivatives, RGMIA Res. Rep. Collect. 1 (1998), 101-111.

[3] P. Cerone, A new Ostrowski type inequality involving integral means over end intervals, Tamkang J. Math. 33(2) (2002), 109-118.

[4] A. Qayyum, M. Shoaib, and I. Faye, On new weighted Ostrowski type inequalities involving integral means over end intervals and application, Turkish J. Anal. Numb. Theory. 3(2) (2015), 61-67.

[5] N. Barnett, S. S. Dragomir, and I. Gomm, A companion for the Ostrowski and the generalised trapezoid inequalities, Math. Comput. Model. 50(1) (2009), 179-187.

[6] H. Budak, S. Erden, and M. Sarikaya, New Weighted Ostrowski Type Inequalities for Mappings Whose nth Derivatives Are of Bounded Variation, Int. J. Anal. Appl. 12(1) (2016), 71-79.

[7] M. Alomari, A companion of Ostrowski's inequality for mappings whose first derivatives are bounded and applications in numerical integration, Transylvanian J. Math. Mech. 4(2) (2012), 103-109. 
[8] M. Alomari, A generalization of companion inequality of Ostrowski's type for mappings whose first derivatives are bounded and applications in numerical integration, Kragujevac J. Math. 36(1) (2012), 77-82.

[9] W. Liu, New Bounds for the Companion of Ostrowski's Inequality and Applications, Filomat. 28 (2014), 167-178.

[10] R. Agarwal, P. Ravi, M. Luo, and R. Raina, On Ostrowski type inequalities, Fasciculi Math. 56(1) (2016), 5-27.

[11] H. Budak, M. Sarikaya, A New Companion of Ostrowski Type Inequalities for Functions of Two Variables With Bounded Variation, Facta Universitatis, Series: Mathematics and Informatics. 31(2) (2016), 447-463.

[12] A. Qayyum, M. Shoaib, A. Matouk, and M. Latif, On new generalized Ostrowski type integral inequalities, Abstr. Appli. Anal. 2014 (2014), Art. ID 275806.

[13] G. Grüss, Uber das Maximum des absoluten Betrages von $1 /(b-a) \int_{a}^{b} f(x) g(x) d x-1 /(b-a)^{2} \int_{a}^{b} f(x) d x \int_{a}^{b} g(x) d x$, Mathematische Zeitschrift. vol. 39(1) (1935), 215-226.

[14] P. L. Čebyŝev, Sur les expressions approximatives des integrales definies par les autres prises entre les mêmes limites, Proc. Math. Soc. Kharkov. 2 (1882), 93-98.

[15] W.G. Alshanti and A. Qayyum, A Note On New Ostrowski Type Inequalities Using a Generalized Kernel. Bull. Math. Anal. Appl. 9(1) (2017), 74-91.

[16] W.G. Alshanti, A. Qayyum and M.A. Majid, Ostrowski Type inequalities by using General Quadratic Kernel. J. Inequal. Spec. Funct. 8(4) (2017), 111-135. 